\title{
EMOTION DYSREGULATION AS A CORE FEATURE OF BORDERLINE PERSONALITY DISORDER
}

\author{
Catherine R. Glenn, MA, and E. David Klonsky, PhD
}

\begin{abstract}
Borderline personality disorder (BPD) is a debilitating mental illness that affects approximately $1-2 \%$ of the general population. Researchers have increasingly come to view emotion dysregulation as a core feature of BPD. The present study examines the relationship between BPD symptomatology and emotion dysregulation using the Difficulties in Emotion Regulation Scale (DERS) in two college samples. BPD symptoms were assessed by self-report (MSI-BPD) in sample 1 and by semistructured interview (SIDP-IV) in sample 2. Results suggest that emotion dysregulation accounts for unique variance in BPD even after controlling for traditional indicators of negative emotionality, including depression, anxiety, and negative affect. Findings support theories regarding the role of emotion dysregulation in BPD and provide directions for future research.
\end{abstract}

Borderline personality disorder (BPD) is a debilitating mental illness characterized by intense negative emotions, identity confusion, impulsive behaviors, and interpersonal instability. Epidemiological studies indicate that BPD affects about $1-2 \%$ of the general population with higher rates, between 10 and 20\%, in patient populations (Torgersen, Kringlen, \& Cramer, 2001; Widiger \& Weissman, 1991). In addition to being impaired in domains of affective, behavioral, and interpersonal functioning (Linehan, 1993), around $10 \%$ of BPD patients attempt suicide, a rate 50 times higher than the general population (American Psychiatric Association, 2001). Due to its destructive course and high rates of suicidality, patients with BPD require more treatment services than other disordered groups (Bender et al., 2001; Zanarini, Frankenburg, Khera, \& Bleichmar, 2001).

Many criteria for BPD in the DSM-IV reflect abnormalities in emotional functioning. For example, affective instability, intense anger, and chronic

From Stony Brook University (C. R. G., E. D. K.).

Address correspondence to E. David Klonsky, PhD, Assistant Professor, Department of Psychology, Stony Brook University; E-mail: E.David.Klonsky@stonybrook.edu 
feelings of emptiness directly reflect aspects of emotion difficulties. Additionally, other BPD criteria, such as self-harm, appear to result from emotion problems. For example, both nonsuicidal self-injury and suicide attempts are often performed to obtain relief from overwhelming, negative emotions (Brown, Comtois, \& Linehan, 2002; Klonsky, 2007). While negative emotionality characterizes many disordered groups, such as dysthymic patients, BPD patients are distinguished by the presence of affective instability in addition to negative emotionality (Conklin, Bradley, \& Westen, 2006). Of all DSM-IV BPD criteria, affective instability appears to best differentiate borderlines from non-borderlines (Clifton \& Pilkonis, 2007).

Not surprisingly, the current and central theory of BPD, Linehan's (1993) biosocial theory, focuses on these emotion difficulties. This theory posits that BPD patients adopt poor coping skills because they are raised in an invalidating environment and have a biological propensity to react emotionally. As a result, BPD patients are characterized by high sensitivity to emotional stimuli, heightened emotional intensity, and slow return to baseline.

Consistent with Linehan's theory of BPD, Lynch et al. (2006) found that BPD patients display increased emotion sensitivity. When asked to recognize emotional facial expressions, BPD patients were faster than controls regardless of the type of emotion. In addition, research using real-time, daily diaries, suggests that BPD patients also vacillate more rapidly between negative and positive emotions (Ebner-Premier et al., 2007).

Further evidence using biological measures of emotion in borderline patients has begun to systematically assess emotional processing and also provide support for emotion hyper-reactivity in BPD. Studies have consistently documented that compared to controls BPD patients have a heightened startle response to unpleasant stimuli (Ebner-Priemer et al., 2005) even when controlling for comorbid diagnoses of anxiety and PTSD (Hazlett et al., 2007). In comparison to controls, BPD patients also exhibit hyperactive amygdala activity when viewing emotional stimuli (Herpertz et al., 2001; Donegan et al., 2003). Taken together these studies support the notion of increased emotion intensity in BPD patients.

This study aims to build upon past research examining the role of emotion difficulties in BPD patients. While great progress has been made in establishing emotion problems as central to our understanding of BPD, past research has not clearly addressed the components of emotion difficulties. Linehan's (1993) theory of BPD suggests at least two features of emotion that may be impaired in BPD: (1) hyper-intensity/reactivity (i.e., emotions that are too strong or triggered too easily), and (2) poor regulation (i.e., deficient control or modulation of the emotional response once it has begun). Researchers have come to increasingly view the second component, emotion dysregulation, as the core feature of BPD (Conklin et al., 2006). Emotion dysregulation is the inability to control and modulate one's affective state to such a degree that emotions can become out of control and override judgment and reason (Linehan \& Heard, 1992; Shedler \& 
Westen, 2004). A preliminary self-report study supports the view that emotion dysregulation drives the symptoms of BPD to a greater extent than high negative emotionality (Yen, Zlotnick, \& Costello, 2002).

The current study assesses the relative contribution of emotion dysregulation and other emotion difficulties to BPD symptomatology. The majority of previous studies have utilized measures that did not disambiguate emotion dysregulation from other aspects of emotion such as reactivity/intensity. For example, in one study, a relationship between scores on the Affect Control Scale and BPD symptoms was interpreted to support an emotion dysregulation theory of BPD (Yen et al., 2002). However, the Affect Control Scale was developed to assess "fear of losing control over one's emotions" (Williams, Chambless, \& Ahrens, 1997, p. 3), which is not equivalent to how one regulates their emotions. In contrast, the present study uses the Difficulties in Emotion Regulation Scale (DERS; Gratz \& Roemer, 2004), which is a more specific, subtle, and comprehensive measure of emotion dysregulation. The DERS assesses six domains of emotion dysregulation, including: nonacceptance of emotion responses (Nonacceptance), difficulties engaging in goal-directed behavior (Goals), impulse control difficulties (Impulse), lack of emotional awareness (Awareness), limited access to emotion regulation strategies (Strategies), and lack of emotional clarity (Clarity). Preliminary research suggests that the DERS has good reliability and predictive validity (Gratz \& Roemer, 2004). In addition, the DERS has shown good correspondence with biological measures of emotion dysregulation, such as respiratory sinus arrhythmia (Crowell et al., 2006).

More specifically, the primary goal of this study is to assess whether emotion dysregulation accounts for unique variance in BPD symptoms over and above traditional measures of negative emotionality, including depression, anxiety, and negative affect. In particular, we are interested in evaluating whether the DERS taps into distinct aspects of emotion difficulties beyond traditional measures that assess emotion reactivity and intensity. Based upon existing conceptualizations of emotion dysregulation in

$\mathrm{BPD}$, we hypothesized that emotion dysregulation, as measured by the DERS, would exhibit a unique association to BPD traits beyond that accounted for by preexisting measures of negative emotionality, and thus provide further evidence that emotion dysregulation represents a distinct and core deficit in BPD.

\section{METHOD}

SAMPLE 1

Participants. The original data set consisted of 273 undergraduate students from lower-level psychology courses. After data screening, 30 participants were removed from the sample: 26 participants were excluded due 
to missing values and 4 due to random responding (i.e., 20 or more consecutive, identical responses). The screened sample included 243 undergraduate students (126 male, 117 female). All participants completed a battery of self-report measures for course credit.

Measures. BPD was measured in this sample using the McLean Screening Instrument for Borderline Personality Disorder (MSI-BPD), a 10-item self-report measure of BPD features (Zanarini et al., 2003). When compared to a validated structured interview, sensitivity, and specificity of the MSI-BPD were both above .90 in young adults.

The Difficulties in Emotion Regulation Scale (DERS; Gratz \& Roemer, 2004) was utilized to evaluate emotion dysregulation. Sample items from the subscales include: When I'm upset, I become angry with myself for feeling that way (Nonacceptance); When I'm upset, I have difficulty concentrating (Goals); When I'm upset, I have difficulty controlling my behaviors (Impulse); When I'm upset, I acknowledge my emotions (Awareness); When I'm upset, I believe that there is nothing I can do to make myself feel better (Strategies); and I have difficulty making sense out of my feelings (Clarity). Each item is rated on "how often the following statements apply to you" on a scale from 1 (almost never) to 5 (almost always).

Depression and anxiety were assessed using the Depression Anxiety Stress Scale (DASS-21; Henry \& Crawford, 2005). The DASS-2 1 is a shortened version of the original 42-item scale (Lovibond \& Lovibond, 1995). Research has confirmed the construct validity of the DASS- 21 in nonclinical samples (Henry \& Crawford, 2005). The short version of the Positive and Negative Affect Scale (PANAS; Watson, Clark, \& Tellegen, 1988) was used to measure negative affect. The PANAS-NA (Negative Affect), a 10item self-report measure, demonstrates good internal consistency (.85), test-retest reliability (.71), and discriminant validity.

\section{SAMPLE 2}

Participants. Participants were 30 students ( 15 male, 15 female) from a college sample recruited for a larger study on the psychophysiology of emotion. All participants provided written consent and completed a semistructured interview and series of self-report measures for psychology course research credit.

Measures. BPD symptomatology was assessed in this sample using the Structured Interview for DSM-IV Personality (SIDP-IV; Pfohl, Blum, \& Zimmerman, 1997). The SIDP-IV is a semistructured interview that assesses each of the 10 DSM-IV personality disorders including borderline personality disorder (Widiger \& Coker, 2002). Reliability and validity of the SIDP has been verified in both non-treatment-seeking and patient populations (Jane, Pagan, Turkheimer, Fiedler, \& Oltmanns, 2006; Pilkonis et al., 1995). Emotion dysregulation, depression, and anxiety were assessed using the same measures as for Sample 1. 


\section{RESULTS}

\section{SAMPLE 1}

The mean score on the MSI-BPD was $3.17(S D=2.58)$. Using a recommended cutoff score of 7 (see Zanarini et al., 2003), 30 participants $(12.3 \%$ of the sample) were likely to meet criteria for a diagnosis of BPD. Table 1 displays the bivariate correlations along with means and standard deviations for the negative emotionality measures and BPD symptoms (MSIBPD). As expected, there was a robust association between BPD symptomatology and the DERS $(r=.54)$. This correlation remained robust $(r=.54)$ even when the affective instability criterion was removed from the BPD total score. Regarding facets of emotion dysregulation, the Strategies and Impulse subscales of the DERS exhibited the largest associations with $\mathrm{BPD}$ ( $r \mathrm{~s}=.55$ and .47 , respectively). BPD was also significantly associated with the other major study variables: depression, anxiety, and negative affect ( $r \mathrm{~s}=.58, .47$, and .41 , respectively).

A series of partial correlations was conducted to measure the unique relationship between emotion dysregulation and BPD symptomatology controlling for depression, anxiety, and negative affect (see Table 2). Findings suggest that the relationship between emotion dysregulation and BPD symptomatology remains significant after controlling for depression, anxiety, and negative affect separately. In addition, after controlling for all negative emotionality variables simultaneously, the relationship between emotion dysregulation and BPD remained significant ( $p r=.24, p<.001)$. Specifically, the emotion dysregulation subscales, Impulse and Strategies, showed the strongest associations with BPD symptomatology when simultaneously controlling for all negative emotionality variables ( $p r s=.26$ and $.24, p<.001$, respectively).

TABLE 1. Correlations Between and Descriptive Statistics for Emotion Variables and BPD-Sample 1

\begin{tabular}{lcrc}
\hline & $\begin{array}{c}\text { BPD } \\
\text { (MSI-BPD) }\end{array}$ & \multicolumn{2}{c}{$\boldsymbol{M}$ (SD) } \\
\hline Emotion Regulation (DERS) & .54 & $81.66(21.45)$ \\
Nonacceptance & .38 & 12.08 & $(5.40)$ \\
Goals & .34 & 14.88 & $(5.16)$ \\
Impulse & .47 & 11.49 & $(4.92)$ \\
Awareness & .03 & 15.33 & $(4.71)$ \\
Strategies & .55 & 16.39 & $(7.12)$ \\
Clarity & .35 & 11.48 & $(3.94)$ \\
Depression & .58 & 4.93 & $(4.71)$ \\
Anxiety & .47 & 4.18 & $(4.04)$ \\
Negative Affect & .41 & 26.63 & $(7.46)$ \\
\hline
\end{tabular}

Note. Correlations above .13 are statistically significant at an alpha level of .05, and above .23 are statistically significant at an alpha level of .001. 


\begin{tabular}{|c|c|c|c|c|}
\hline & $\begin{array}{l}\text { Depression } \\
\text { Controlled }\end{array}$ & $\begin{array}{c}\text { Anxiety } \\
\text { Controlled }\end{array}$ & $\begin{array}{c}\text { Negative } \\
\text { Affect } \\
\text { Controlled } \\
\end{array}$ & $\begin{array}{l}\text { All Three } \\
\text { Variables } \\
\text { Controlled }\end{array}$ \\
\hline Emotion Dysregulation & .30 & .42 & .45 & .24 \\
\hline Nonacceptance & .18 & .27 & .31 & .15 \\
\hline Goals & .18 & .25 & .25 & .13 \\
\hline Impulse & .31 & .36 & .40 & .26 \\
\hline Awareness & -.06 & -.01 & .03 & -.04 \\
\hline Strategies & .29 & .44 & .46 & .24 \\
\hline Clarity & .15 & .25 & .30 & .14 \\
\hline
\end{tabular}

Note. Correlations above .13 are statistically significant at an alpha level of .05, and above .23 are statistically significant at an alpha level of .001 .

\section{SAMPLE 2}

Thirty percent of the sample met multiple criteria (two or more) for BPD on the SIDP-IV (Pfohl et al., 1997), including one participant who qualified for a BPD diagnosis. Table 3 displays the bivariate correlations along with means and standard deviations for the negative emotionality measures and BPD symptoms. There was a robust association between BPD symptomatology and emotion dysregulation $(r=.64)$. This correlation remained robust $(r=.65)$ even when the affective instability criterion was removed from the BPD total score. As in Sample 1, the strongest associations between emotion dysregulation and BPD symptoms were for the Impulse and Strategies subscales of the DERS ( $r \mathrm{~s}=.76$ and .67 , respectively). BPD symptomatology was also significantly associated with the other major study variables: depression and anxiety $(r \mathrm{~s}=.57$ and .58 , respectively) (negative affect was not measured in sample 2).

As in Sample 1, a series of partial correlations was conducted to measure the unique relationship between emotion dysregulation and BPD

TABLE 3. Correlations Between and Descriptive Statistics for Emotion Variables and BPD-Sample 2

\begin{tabular}{lccc}
\hline & $\begin{array}{c}\text { BPD } \\
\text { (SIDP-IV) }\end{array}$ & \multicolumn{2}{c}{$\boldsymbol{M}$ (SD) } \\
\hline Emotion Regulation (DERS) & .64 & 76.53 & $(21.76)$ \\
Nonacceptance & .45 & 10.33 & $(2.99)$ \\
Goals & .49 & 14.40 & $(4.92)$ \\
Impulse & .76 & 11.03 & $(4.90)$ \\
Awareness & .18 & 13.57 & $(4.20)$ \\
Strategies & .67 & 16.57 & $(6.79)$ \\
Clarity & .34 & 10.63 & $(3.68)$ \\
Depression & .57 & 3.53 & $(4.23)$ \\
Anxiety & .58 & 2.83 & $(3.04)$ \\
\hline
\end{tabular}

Note. Correlations above .38 are statistically significant at an alpha level of .05, above .44 are statistically significant at an alpha level of .01, and above .56 are statistically significant at an alpha level of .001. 
symptomatology controlling for depression and anxiety (see Table 4). Findings suggest that the relationship between emotion dysregulation and BPD symptomatology remains significant after controlling for depression $(r=$ $.42, p<.05)$ and anxiety $(r=.50, p<.01)$, separately. After controlling for both depression and anxiety simultaneously, the relationship between emotion dysregulation and BPD symptoms was marginally significant ( $r=$ $.35, p=.07$ ). Partial correlations were statistically significant for the emotion dysregulation Impulse subscale $(r=.55, p<.01)$ and Strategies subscale $(r=.37, p=.05)$.

\section{DISCUSSION}

The present study examines the relationship between emotion dysregulation and BPD symptomatology. More specifically, the purpose was to examine whether emotion dysregulation explains unique variance in BPD over and above traditional measures of negative emotionality, including depression, anxiety, and negative affect. Results suggest that emotion dysregulation exhibits a robust and unique relationship with BPD symptomatology. This association was observed when measuring BPD symptoms either by self-report or semistructured interview. Emotion regulation difficulties resulting from impulse control difficulties and limited access to emotion regulation strategies exhibited the strongest relationship to BPD.

Findings support theories suggesting that emotion dysregulation is a core feature of BPD. In addition, results suggest that the assessment of emotion dysregulation adds unique information to our understanding of BPD symptomatology beyond that assessed by other measures of negative emotionality. Further, the present study supports the validity of the Difficulties in Emotion Regulation Scale (DERS) as a measure of emotion dysregulation that captures important information not tapped by other valid measures of emotion dysfunction.

The present study helps establish emotion dysregulation as a distinct and important feature of BPD. However, the study also has several limita-

\begin{tabular}{lccc}
$\begin{array}{c}\text { TABLE 4. Partial Correlations Between Emotion Dysregulation } \\
\text { and BPD Symptomatology Controlling for Traditional Indices } \\
\text { of Negative Emotionality-Sample 2 }\end{array}$ \\
\hline & $\begin{array}{c}\text { Depression } \\
\text { Controlled }\end{array}$ & $\begin{array}{c}\text { Anxiety } \\
\text { Controlled }\end{array}$ & $\begin{array}{c}\text { Both } \\
\text { Variables } \\
\text { Controlled }\end{array}$ \\
\hline & .42 & .50 & .35 \\
\hline Emotion Dysregulation & .15 & .32 & .12 \\
Nonacceptance & .27 & .36 & .23 \\
Goals & .61 & .64 & .55 \\
Impulse & -.01 & .21 & .08 \\
Awareness & .46 & .51 & .37 \\
Strategies & .16 & .16 & .06 \\
Clarity &
\end{tabular}

Note. Correlations above .38 are statistically significant at an alpha level of .05, and above .56 are statistically significant at an alpha level of .001. 
tions and future research is needed. One limitation of the current study is the nature of the sample, which was drawn from a college population. Future research should replicate findings in larger samples and with clinical populations. In addition, emotion dysregulation and other emotion variables were assessed using self-report measures. Future research should utilize alternative measures of emotion and emotion regulation, such as daily diary and physiological methodologies which are not subject to the same biases as retrospective self-reports. Lastly, future research should identify which aspects of emotion dysregulation are most amenable to psychiatric intervention and best predict course and prognosis.

\section{REFERENCES}

American Psychiatric Association. (2001). Practice guideline for the treatment of patients with borderline personality disorder-introduction. American Journal of Psychiatry, 158.

Bender, D. S., Dolan, R. T., Skodol, A. E., Sanislow, C. A., Dyck, I. R., McGlashan, T. H., et al. (2001). Treatment utilization by patients with personality disorders. American Journal of Psychiatry, 158, 295-302.

Brown, M. Z., Comtois, K. A., \& Linehan, M. M. (2002). Reasons for suicide attempts and nonsuicidal self-injury in women with borderline personality disorder. Journal of Abnormal Psychol ogy, 111, 198-202.

Clifton, A., \& Pilkonis, P. A. (2007). Evidence for a single latent class of diagnostic and statistical manual of mental disorders borderline personality pathology. Comprehensive Psychiatry, 48, 70-78.

Conklin, C. Z., Bradley, R., \& Westen, D. (2006). Affect regulation in borderline personality disorder. The Journal of Nervous and Mental Disease, 194, 69-77.

Crowell, S. E., Beauchaine, T. P., Marsh, P., Shannon, K. E., Neuhaus, E., \& Chipman-Chacon, J. (2006). Correspondence between psychophysiological and self-report measures of emotion regulation in a pre-adolescent sample. Poster presented at the annual meeting of the Society for Psychophysiological Research, Vancouver, CA.

Donegan, N. H., Sanislow, C. A., Blumberg, H. P., Fulbright, R. K., Lacadie, C., Skudlarski, P., et al. (2003). Amygdala hyperreactivity in borderline personal- ity disorder: Implications for emotional dysregulation. Biological Psychiatry, 54, 1284-1293.

Ebner-Priemer, U. W., Badeck, S., Beckmann, C., Wagner, A., Feige, B., Weiss, I., Lieb, K., \& Bohus, M. (2005). Affective dysregulation and dissociative experience in female patients with borderline personality disorder: A startle response study. Journal of Psychiatric Research, 39, 85-92.

Ebner-Priemer, U. W., Kuo, J., Kleindienst, N., Welch, S. S., Reisch, T., Reinhard, I., et al. (2007). State affective instability in borderline personality disorder assessed by ambulatory monitoring. Psychological Medicine, 37, 961-970.

Gratz, K. L., \& Roemer, L. (2004). Multidimensional assessment of emotion regulation and dysregulation: Development, factor structure, and initial validation of the difficulties in emotion regulation scale. Journal of Psychopathology and Behavioral Assessment, 26, 41-54.

Hazlett, E. A., Speiser, L. J., Goodman, M., Roy, M., Carrizal, M., Wynn, J. K., et al. (2007). Exaggerated affect-modulated startle during unpleasant stimuli in borderline personality disorder. Biological Psychiatry, 62, 250-255.

Henry, J. D., \& Crawford, J. R. (2005). The short-form version of the depression anxiety stress scale (DASS-21): Construct validity and normative data in a large non-clinical sample. British Journal of Clinical Psychology, 44, 227239.

Herpertz, S. C., Dietrich, T. M., Wenning, B., Krings, T., Erberich, S. G., Willmes, K., 
Thron, A., \& Sass, H. (2001). Evidence of abnormal amygdala functioning in borderline personality disorder: A functional MRI study. Biological Psychiatry, 50, 292-298.

Jane, J. S., Pagan, J. L., Turkheimer, E., Fiedler, E. R., \& Oltmanns, T. F. (2006). The interrater reliability for the Structured Interview for DSM-IV Personality. Comprehensive Psychiatry, 47, 368-375.

Klonsky, E. D. (2007). The functions of deliberate self-injury. A review of the evidence. Clinical Psychology Review, 27, 226-239.

Linehan, M. M. (1993). Skills-training manual for treatment of borderline personality disorder. New York: Guilford.

Linehan, M. M., \& Heard, H. L. (1992). Dialectical behavior therapy for borderline personality disorder. In J. F. Clarkin, E. Marziali, H. Munroe-Blum (Eds), Borderline personality disorder: Clinical and empirical perspectives (pp. 248-267). New York: Guilford Press.

Lovibond, S. H., \& Lovibond, P. F. (1995). Manual for the depression anxiety stress scales, (2nd ed.). Sydney, Australia: Psychology Foundation of Australia.

Lynch, T. R., Rosenthal, M. Z., Kosson, D. S., Cheavens, J. S., Lejuez, C. W., \& Blair, R.J.R. (2006). Heightened sensitivity to facial expressions of emotion in borderline personality disorder. Emotion, 6, 647-655.

Pfohl, B., Blum, N., \& Zimmerman, M. (1997). Structured interview for DSM-IV personality. Washington DC: American Psychiatric Press.

Pilkonis, P. A., Heape, C. L., Proietti, J. M., Clark, S. W., McDavid, J. D., \& Pitts, T. E. (1995). The reliability and validity of two structured diagnostic interviews for personality disorders. Archives of General Psychiatry, 52, 1025-1033.

Shedler, J., \& Westen, D. (2004). Refining personality disorder diagnosis: Integrating science and practice. American Journal of Psychiatry, 161, 1350-1365.

Torgersen, S., Kringlen, E., \& Cramer, V. (2001). The prevalence of personality disorders in a community sample. Archives of General Psychiatry, 58, 590596.

Watson, D., Clark, L. A., \& Tellegen, A. (1988). Development and validation of brief measures of positive and negative affect: The PANAS scales. Journal of Personality and Social Psychology, 54, 1063-1070.

Widiger, T. A., \& Coker, L. A. (2002). Assessing personality disorders. In J. N. Butcher (Ed.), Clinical personality assessment. Practical approaches (2nd ed., pp. 380-394). New York: Oxford University Press.

Widiger, T. A., \& Weissman, M. M. (1991). Epidemiology of borderline personality disorder. Hospital \& Community Psychiatry, 42, 1015-1021.

Williams, K. E., Chambless, D. L., \& Ahrens, A. (1997). Are emotions frightening? An extension of the fear of fear construct. Behaviour Research and Therapy, 35, 239-248.

Yen, S., Zlotnick, C., \& Costello, E. (2002). Affect regulation in women with borderline personality disorder traits. The Journal of Nervous and Mental Disease, 190, 693-696.

Zanarini, M. C., Frankenburg, F. R., Khera, G. S., \& Bleichmar, J. (2001). Treatment histories of borderline inpatients. Comprehensive Psychiatry, 42, 144-150.

Zanarini, M. C., Vujanovic, A. A., Parachini, E. A., Boulanger, J. L., Frankenburg, F. R., \& Hennen, J. (2003). A screening measure for BPD: The McLean screening instrument for borderline personality disorder (MSI-BPD). Journal of Personality Disorders, 17, 568-573. 